\title{
Chronic disease management items in general practice: a population-based study of variation in claims by claimant characteristics
}

\author{
Kirsty A Douglas, Laurann E Yen, Rosemary J Korda, Marjan Kljakovic and Nicholas J Glasgow
}

$\mathrm{H}$ ealth policies globally have emphasised better coordination and planning of health care as key components in improving outcomes for people with chronic illness. ${ }^{1-3}$ Lack of integration and coordination continue to be identified as key barriers to good management. ${ }^{4}$

Most primary medical care in Australia is provided on a fee-for-service basis, paid by Medicare, the universal health insurance scheme, according to the Medical Benefits Schedule (MBS) of payments, sometimes with patient copayments. The introduction of the Enhanced Primary Care (EPC) package in 1999 added specific items to the MBS to fund planning and coordination of complex care. $^{2}$

Barriers to the use of EPC items have been identified $^{5-7}$ and debated. ${ }^{8,9}$ Yet although Medicare claims for chronic disease (CD) items have increased from 1.1 million claims, at a cost of $\$ 117$ million, in the financial year $2005-06,{ }^{10}$ to over 3 million claims costing $\$ 311$ million in 2009-10, ${ }^{11}$ there has been limited published work evaluating their use or effectiveness. ${ }^{12-15}$

This study describes variation in claims for MBS CD items by sociodemographic and health characteristics in people with heart disease, diabetes or asthma.

\section{METHODS}

We used data from the 45 and Up Study, a large-scale cohort study involving men and women aged 45 years and over from New South Wales, Australia. ${ }^{16}$

Participants in the study were randomly sampled from the Medicare database, which provides virtually complete coverage of the Australian population. They joined the study by completing a baseline questionnaire. ${ }^{17}$ All participants gave signed consent for follow-up and linkage to a range of health databases. We used data on the first 102934 participants enrolled in the study, who completed the baseline questionnaire between January 2006 and July 2008. We selected participants with heart disease, diabetes or asthma, based on their response to the question "Has a doctor ever told you that

\section{ABSTRACT}

Objective: To describe how Medical Benefits Schedule (MBS) chronic disease (CD) item claims vary by sociodemographic and health characteristics in people with heart disease, asthma or diabetes.

Design, setting and participants: A cross-sectional analysis of linked unit-level MBS and survey data from the first 102934 participants enrolled in the 45 and Up Study, a large-scale cohort study in New South Wales, who completed the baseline survey between January 2006 and July 2008.

Main outcome measure: Claim for any general practitioner CD item within 18 months before enrolment, ascertained from MBS records.

Results: The proportion of individuals making claims for MBS CD items was $18.5 \%$ for asthma, 22.3\% for heart disease, and $44.9 \%$ for diabetes. Associations between participant characteristics and a claim for a CD item showed similar patterns across the three diseases. For heart disease and asthma, people most likely to claim a CD item were women, older, of low income and education levels, with multiple chronic conditions, fair or poor self-rated health, obesity and low physical activity levels. The pattern of claims was slightly different for participants with diabetes in that there was no significant association with number of chronic conditions, smoking or physical activity.

Conclusions: Many individuals with self-reported CD do not claim CD items. People with diabetes and individuals with greatest need based on health, socioeconomic and lifestyle risk factors are the most likely to claim CD items.

MJA 2011; 195: 198-202

you have ..." followed by a list of 14 common chronic or complex conditions, including heart disease, diabetes, and asthma (listed separately from January 2007). Survey data for each participant were linked to MBS data, which included records of every medical service claimed by study participants since July 2004. These records contain MBS item numbers and the date on which each service was received. All participants were enrolled at least 18 months after June 2004, enabling an adequate time period to capture claims for a CD management service.

The outcome variable was the claim for any general practitioner $C D$ item number (MBS Items 721-732) within 18 months before enrolment in the study, which was coded as a dichotomous variable ( $1=$ yes; $0=$ no). For patients with asthma, the outcome variable was also coded as 1 if the participant had claimed an asthma annual cycle of care service (MBS Items 2546-2559 and 2664-2677). For patients with diabetes, the outcome variable was also coded as 1 if the participant had claimed a diabetes annual cycle of care service (MBS Items 2517-2526 and 2620-2635). Sociodemographic and health-related exposure variables were based on self-reported data collected on the baseline survey. The main sociodemographic variables of interest were sex, age, area of residence, education and household income, and the health variables of interest were overall self-rated health, body mass index, smoking, physical activity, alcohol consumption and other chronic conditions from the list of those available (Box). Other variables (data not shown) included country of birth (Australia, New Zealand, elsewhere), marital status (married, de facto, not married), area-based socioeconomic status (SES) (quintiles of disadvantage based on Socio-Economic Indexes for Areas, ${ }^{18}$ derived from postcode of residence), employment status (employed/ retired/not employed) and Health Care Card (yes/no).

The strength of association between each sociodemographic or health variable and a 
CD item claim for people reporting heart disease, asthma and diabetes was estimated using logistic regression, with the results presented as odds ratios adjusted for age and sex. Analyses were conducted using Stata, version 11.1 (StataCorp, College Station, Tex, USA). Two sensitivity analyses were done to examine potential biases. The first analysis involved excluding participants who were diagnosed within the 18 months before enrolment, to limit the analysis to those who were potentially eligible for a CD item for the full 18 months. The second analysis excluded participants who enrolled before January 2007, to limit the analysis to the period after introduction of the new CD items (in July 2005).

\section{Ethics approval}

The 45 and Up Study has approval from the University of New South Wales Human Research Ethics Committee. We obtained approval from the Australian National University Human Research Ethics Committee for our study.

\section{RESULTS}

After excluding those for whom Medicare data were not yet linked at the time of this study $(1044,1 \%)$, there were 12545 people identified with heart disease, 7659 with asthma and 9113 with diabetes.

Among participants with heart disease, just over a fifth (22.3\%) claimed at least one $\mathrm{CD}$ item in the 18 months before their enrolment in the study. Associations between patient characteristics and a claim for a CD item were significant for all the factors except smoking (Box). People most likely to claim a CD item were women; older (aged above 54 years); of lower SES (ie, for income and education); living in an inner regional area (least likely in remote areas); in relatively poor health (ie, multiple chronic conditions, fair or poor self-rated health, obese, low physical activity levels); and nondrinkers. Claimants were also less likely to have private health insurance or a DVA (Department of Veterans' Affairs) treatment card.

Among people with asthma, less than one in five $(18.5 \%)$ claimed a CD item in the 18 months before their enrolment in the study, and only $1.5 \%$ claimed an asthma annual cycle of care service. Associations between patient characteristics and claims for a CD item showed a very similar pattern to that of people with heart disease (Box). However, among people with asthma (unlike among those with heart disease), past smokers and current smokers were more likely to have claimed a CD item than participants who had never smoked.

Among participants with diabetes, almost half (44.9\%) claimed a CD item, with $23.1 \%$ claiming a diabetes annual cycle of care service. The pattern of service use with respect to sociodemographic and health characteristics was slightly different compared with the patterns found among people with heart disease and asthma. Participants with diabetes who lived in remote areas were more likely to claim a CD item than those living in major cities, and there was no association with number of chronic conditions, smoking or physical activity. People most likely to claim a CD item were women; aged 65-74 years; of lower SES (income and education); obese; without a DVA card or private insurance; and non-drinkers (Box).

In addition to the results shown in the Box, people with heart disease, asthma or diabetes were significantly less likely to claim a CD item if they lived in areas of least disadvantage, were currently employed, and did not have a Health Care Card. These findings, like those above, show a greater probability of a CD claim among people of low SES. Among people with heart disease, those born outside Australia or New Zealand were significantly more likely to claim a CD item than those born in Australia or New Zealand, while the reverse was true among those with diabetes. Among people with heart disease or asthma, those who were not married were significantly more likely to claim an item than those who were married or in a de facto relationship (data available on request).

We also separately examined a subset of the CD items - for Team Care Arrangements (TCAs) (Items 723 and 727) — and found that about half of those with any CD item had claimed a TCA item (data available on request). The pattern and strength of association between these TCA items and sociodemographic and health characteristics were very similar to those reported above for any $C D$ item. The two sensitivity analyses showed results to be essentially unchanged after excluding participants who were diagnosed within the 18 months before enrolment, and after excluding only those who enrolled before January 2007.

\section{DISCUSSION}

Our study is the first to link unit-level survey and MBS data to examine claims for MBS CD items. We have shown that while the majority of people with heart disease, asthma or diabetes do not claim CD items, such items are most likely to be claimed by individuals of low income, low educational attainment, and poor health. Individuals with different lifestyle risk factors also have different claim patterns. High body mass index, and in some cases levels of physical activity and smoking, are also associated with an increased likelihood of a claim.

Strengths of our study include the large number of participants and the ability to link individual survey data to MBS records. The study examines CD item claims made between 7 and 9 years after the EPC package was launched, at least 2 years after changes were made in item descriptors, and following significant government and Division of General Practice promotion. Thus the study reflects the use of the items in a mature policy environment. A limitation is that no case note review of care was undertaken and some individuals may have had formal CD management plans created but never claimed against the item number. Another limitation is that all exposure data were selfreported. In particular, self-report of morbidity has well known methodological limitations, and the simple enumeration of chronic conditions from a restricted list, with no assessment of severity or time since diagnosis, is a crude measure of comorbidity.

There are no previous studies with which we can directly compare our results. Our finding that women and those with multiple chronic conditions are the most likely to claim CD items is similar to that of a recent clinically based study on TCAs, ${ }^{15}$ and our finding that people of lower SES are more likely to claim CD items than those with higher SES is consistent with a study based on MBS data aggregated by SES of postcode of general practice. ${ }^{12,19}$ This suggests that these items are claimed by those most in need. In contrast to aggregate-level studies, ${ }^{19}$ but similar to the individual level study of TCAs, ${ }^{15}$ we found that people (with asthma and heart disease at least) had a significantly decreased likelihood of claiming for a CD item in remote areas compared with major cities. As access to Medicare rebates for allied health services is a driver of some of the CD items, ${ }^{13}$ the relative paucity of available allied health services in remote areas may go some way to explaining this finding.

There were significant differences in the patterns of MBS CD item use across the three CDs. This suggests that GPs discriminate, perhaps appropriately, between condi- 


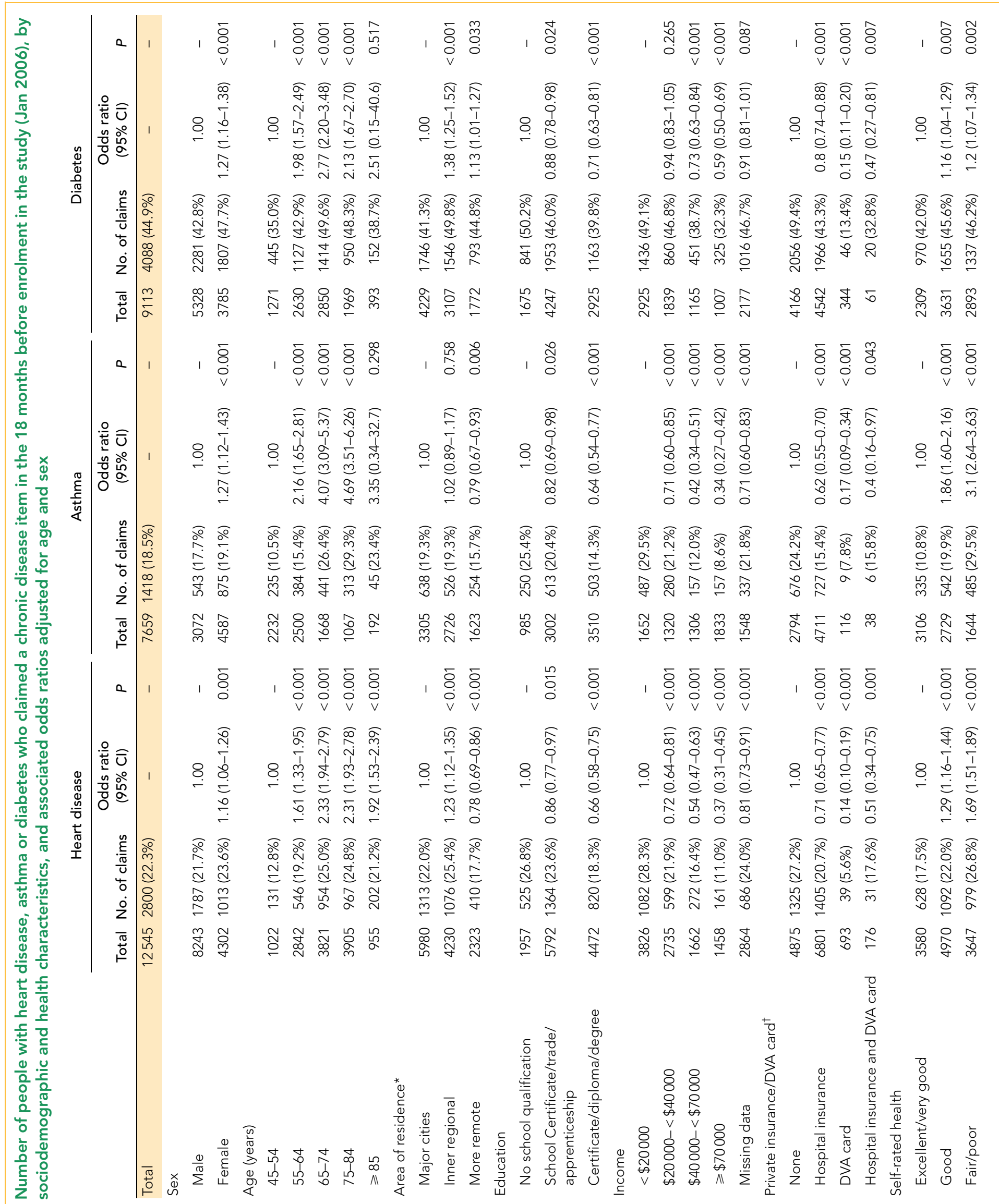




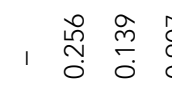

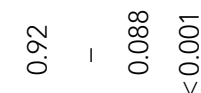

1 $\frac{\pi}{\sigma} \frac{\hat{\sigma}}{0}$

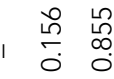

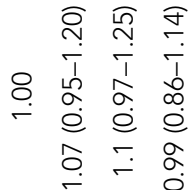

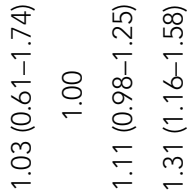

응 ㅇํㅇ

ป

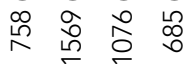

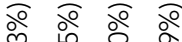

誌过 过

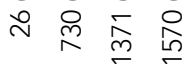

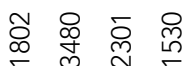

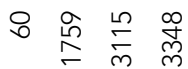

\begin{tabular}{lll}
$\overline{8}$ & $\overline{8}$ & $\overline{8}$ \\
\hline & 0 & 0 \\
0 & 0 \\
V & v
\end{tabular}

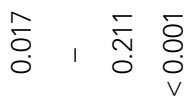

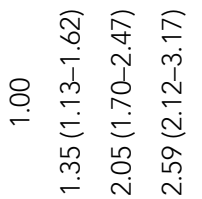

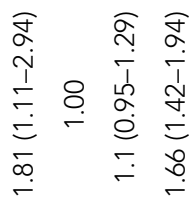

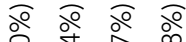

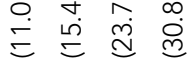

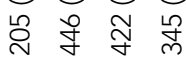

ㅇํㅇ ㅇํㅇ ㅇํํ

ปู

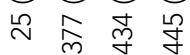

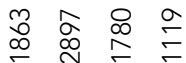

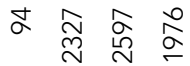

$\begin{array}{lll}5 & 5 & \overline{8} \\ \circ & 0 & 0 \\ \circ & 0 & 0\end{array}$

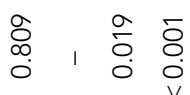

f

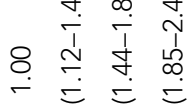

$=\bar{m}=$

๗઼

ㅇํํ ㅇํํ ㅇํㅇ

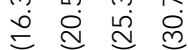

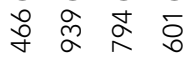

ชิ

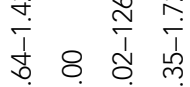

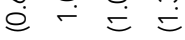

ำ ๖ ก

ㅇำ $\widehat{\circ}$

œ

$=\underset{\mathbb{d}}{\mathbb{d}}$

담 잉

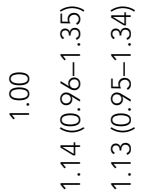

๙ิे ㅇํำ

峁 过

崩

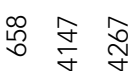

I $\stackrel{0}{\frac{\sigma}{\sigma}} \overline{8}$

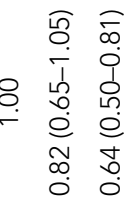

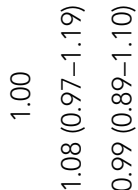

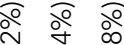

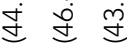

సิ ๘

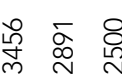

m

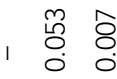

ㅇํㅇ ㅇํㅇ ㅇํㅇ

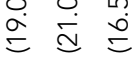

을 ก

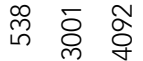

ํํำ ำ

1 0

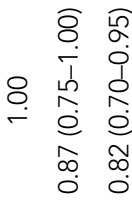

ㅇํㅇ ㅇํㅇ

¿े

年

๗ָׁ

$\begin{array}{lll} & \infty & 0 \\ 0 & 0 & 0 \\ 0 & 0\end{array}$

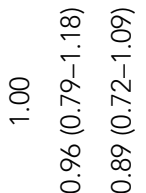

ㅇํㅇ ๙ ๙

ปู ญ

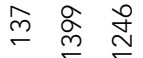

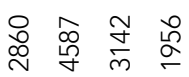

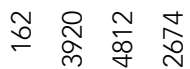

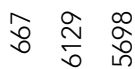

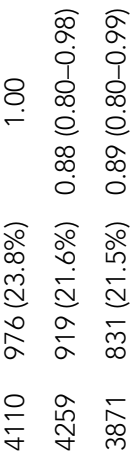

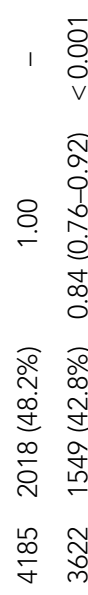

$\begin{array}{ll}\bar{\alpha} & \bar{\delta} \\ 0 & \\ \vee & 0\end{array}$
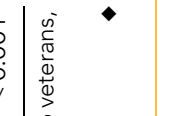

tions when they consider making a $C D$ management plan. For patients with diabetes, the proportion of patients who claimed at least one $\mathrm{CD}$ item was nearly double that for people with either heart disease or asthma. The reasons for this greater use may include the emphasis given to diabetes management in Division of General Practice education and practice support programs, and the influence of accepted best practice guidelines for management and monitoring in diabetes. ${ }^{20}$ It may also reflect the fact that the original EPC items were designed around multidisciplinary practice, and diabetes management best practice explicitly involves other specified professionals. ${ }^{20,21}$ The relatively low proportion of participants with asthma who claimed an item may reflect the fact that asthma is not necessarily a current problem for many participants.

Many individuals with chronic conditions do not claim CD management items, but those who appear to have greatest need, based on health, socioeconomic and lifestyle risk factors, are the most likely to claim them. Further, CD items are claimed most for individuals with diabetes, where the evidence to support structured and multidisciplinary care is strongest. This suggests that Australian GPs are more likely to adopt policy when it is supported by good evidence. Further studies assessing whether MBS CD items are associated with improvements in health outcomes are needed.

\section{ACKNOWLEDGEMENTS}

This research was carried out as part of the Serious and Continuing IIIness Policy and Practice Study, with the support of an National Health and Medical Research Council program grant to the Menzies Centre for Health Policy. This research was completed using data collected through the 45 and Up Study (http://www.45andUp.org.au). The 45 and Up Study is managed by the Sax Institute in collaboration with major partner Cancer Council New South Wales and partners the National Heart Foundation (NSW Division); NSW Health; beyondblue: the national depression initiative; Ageing, Disability and Home Care, Department of Human Services NSW; and UnitingCare Ageing. We thank the many thousands of people participating in the 45 and Up Study.

\section{COMPETING INTERESTS}

None relevant to this article declared (ICMJE disclosure forms completed).

\section{AUTHOR DETAILS}

Kirsty A Douglas, DipRACOG, MD, FRACGP, Research Fellow ${ }^{1}$

Laurann E Yen, BSc, MPsych, Research Fellow ${ }^{2}$ 
Rosemary J Korda, BAppSci, MAppSci, PhD, Research Fellow ${ }^{3}$

Marjan Kljakovic, MB ChB, FRNZCGP, PhD,

Professor of General Practice ${ }^{4}$

Nicholas J Glasgow, BHB, MB ChB, MD, Dean, ANU Medical School ${ }^{4}$

1 Australian Primary Health Care Research Institute, Australian National University,

Canberra, ACT.

2 Menzies Centre for Health Policy, Australian National University, Canberra, ACT.

3 National Centre for Epidemiology and Population Health and Australian Centre for Economic Research on Health, Australian National University, Canberra, ACT.

4 Australian National University Medical School, Australian National University, Canberra, ACT.

Correspondence: laurann.yen@anu.edu.au

\section{REFERENCES}

1 Wagner EH, Austin BT, Michael Von K. Organizing care for patients with chronic illness. Milbank $Q$ 1996; 74: 511-544

2 Australian Government Department of Health and Ageing. MBS Primary Care Items. History of key MBS primary health care initiatives 19992010. http://www.health.gov.au/internet/main/ publishing.nsf/Content/mbsprimarycare-History (accessed Jul 2011).

3 National Health Priority Action Council. National Chronic Disease Strategy. Canberra: Australian Government Department of Health and Ageing, 2006.

4 Yen L, Gillespie J, Jeon Y-H, et al. Health professionals, patients and chronic illness pol- icy: a qualitative study. Health Expectations 2011; 14: 10-20.

5 Blakeman TM, Harris MF, Comino EJ, Zwar NA. Evaluating general practitioners' views about the implementation of the Enhanced Primary Care Medicare items. Med J Aust 2001; 175: 9598.

6 Zwar NA, Comino EJ, Hasan I, Harris MF. General practitioner views on barriers and facilitators to implementation of the Asthma 3+ Visit Plan. Med J Aust 2005; 183: 64-67.

7 Mitchell GK, Tieman JJ, Shelby-James TM. Multidisciplinary care planning and teamwork in primary care. Med J Aust 2008; 188 (8 Suppl): S61-S64.

8 Harris MF, Chan BC, Dennis SM. Coordination of care for patients with chronic disease. Med J Aust 2009; 191: 85-86.

9 Hartigan PA, Soo TM, Kljakovic M. Do Team Care Arrangements address the real issues in the management of chronic disease? Med J Aust 2009; 191: 99-100.

10 Medicare Australia. Statistics. Medicare Item Reports for item numbers 721, 723, 729, 731 and 732 for 2005-06 by count and by expenditure. https://www.medicareaustralia.gov.au/statistics/mbs_item.shtml (accessed Jan 2011).

11 Medicare Australia. Statistics. Medicare Item Reports for item numbers 721, 723, 729, 731 and 732 for $2009-10$ by count and by expenditure. https://www.medicareaustralia.gov.au/statistics/mbs_item.shtml (accessed Jan 2011).

12 Wilkinson D, McElroy $H$, Beilby J, et al. Are socio-economically disadvantaged Australians making more or less use of the Enhanced Primary Care Medicare Benefit Schedule item numbers? Aust Health Rev 2003; 26: 43-49.
13 Shortus TD, McKenzie SH, Kemp LA, et al. Multidisciplinary care plans for diabetes: how are they used? Med J Aust 2007; 187: 78-81.

14 Zwar NA, Hermiz O, Comino EJ, et al. Do multidisciplinary care plans result in better care for patients with type 2 diabetes? Aust Fam Physician 2007; 36: 85-89.

15 Harris MF, Jayasinghe UW, Taggart JR, et al. Multidisciplinary Team Care Arrangements in the management of patients with chronic disease in Australian general practice. Med J Aust 2011; 194: 236-239.

16 Banks E, Redman S, Jorm L, et al. Cohort profile: the 45 and Up Study. Int J Epidemiol 2008; 37: 941-947.

17 Sax Institute. The 45 and Up Study. Study materials and publications. http:// www. 45andup.org.au/studymaterials.aspx? page $=$ studymaterials (accessed Jan 2011).

18 Pink B. Socio-Economic Indexes for Areas (SEIFA) - Technical Paper 2006. Canberra: Australian Bureau of Statistics, 2008. (ABS Cat. No. 2039.0.55.001.)

19 Wilkinson D, McElroy H, Beilby J, et al. Variation in levels of uptake of enhanced primary care item numbers between rural and urban settings, November 1999 to October 2001. Aust Health Rev 2002; 25: 123-130.

20 Harris P, Mann L, Bolger-Harris H, et al, editors. Diabetes management in general practice. Guidelines for type 2 diabetes. 16th ed. Canberra: Diabetes Australia, 2010.

21 Colagiuri R, Girgis S, Eigenmann C, Griffiths R. National evidence based guideline for patient education in type 2 diabetes. Canberra: Diabetes Australian and the National Health and Medical Research Council, 2009.

(Received 17 Feb 2011, accepted 7 Jul 2011) 